\title{
Impact of anti-CD20 monoclonal antibodies on serologic response to BNT162b2 vaccine in B-cell Non-Hodgkin's lymphomas
}

\author{
Francesco Marchesi $\mathbb{D}^{1 凶}$, Fulvia Pimpinelli ${ }^{2}$, Diana Giannarelli ${ }^{3}$, Livia Ronchetti ${ }^{4}$, Elena Papa ${ }^{1}$, Paolo Falcucci ${ }^{1}$, Martina Pontone ${ }^{2}$, \\ Enea Gino Di Domenico ${ }^{2}$, Simona di Martino ${ }^{5}$, Valentina Laquintana ${ }^{5}$, Chiara Mandoj ${ }^{6}$, Laura Conti ${ }^{6}$, Iole Cordone ${ }^{6}$, Antonia La Malfa ${ }^{7}$, \\ Caterina Viggiani ${ }^{1}$, Daniela Renzi ${ }^{1}$, Francesca Palombi ${ }^{1}$, Atelda Romano ${ }^{1}$, Francesco Pisani ${ }^{1}$, Svitlana Gumenyuk ${ }^{1}$, Ornella Di Bella ${ }^{8}$, \\ Branka Vujovic ${ }^{8}$, Aldo Morrone ${ }^{9}$, Gennaro Ciliberto ${ }^{10}$, Fabrizio Ensoli ${ }^{2}$ and Andrea Mengarelli ${ }^{1}$
}

(C) The Author(s), under exclusive licence to Springer Nature Limited 2021

Leukemia (2022) 36:588-590; https://doi.org/10.1038/s41375-021-01418-8

\begin{abstract}
To the Editor:
Patients with hematological malignancies are at risk of severe COVID-19 and candidate to priority vaccination policies, preferably with a mRNA vaccine. In light of ethical considerations and strict indications of Health Authorities (HA), also patients with ongoing lymphodepleting therapy underwent vaccination. In Italy, the national plan against COVID-19 required to proceed as soon as possible with the vaccination of hematological patients in treatment with immunosuppressive or myelosuppressive drugs or within six months from the end of such treatment, and of stem cell transplanted patients after three months from transplant. Lymphoid cancers have been identified as being particularly at risk of inadequate antibody response to anti-SARS-CoV-2 vaccines. In patients with chronic lymphocytic leukemia (CLL), the response is markedly impaired and affected by disease activity and treatment $[1,2]$. Multiple myeloma patients on anti-CD38 antibody therapy also respond partially and weakly [3]. The first observations on immunogenicity of anti-SARS-CoV-2 vaccines in patients with B-cell Non-Hodgkin's Lymphoma (B-NHL) indicate that humoral response to BNT162b2 is deeply impaired in subjects undergoing anti-B cells monoclonal antibodies (moAbs) [4-6]. Such detrimental effect is not surprising, considering that a postponement to six months after therapy suspension has been suggested in patients exposed to anti-CD20 and anti-CD22 moAbs, anti-CD19 bispecific antibody and chimeric antigen receptor T-cells in vaccination settings other than COVID-19 $[7,8]$. According to our mono-centric prospective cohort study formally approved by the IRCCS Central Ethical Committee of Regione Lazio in January 2021 (Prot. N-1463/21), herein we present data on the serologic response to BNT162b2 (two intramuscular injections of $30 \mu \mathrm{g}$ per dose three weeks apart) in 68 consecutive
\end{abstract}

patients with B-NHL on anti-CD20-based therapy (rituximab or obinutuzumab) either ongoing or previously administered, with the primary objective to explore the impact of anti-CD20 moAbs on vaccine immunogenicity and identify the time interval (TI) free from an anti-CD20 administration required for mitigating such effect. Anti SARS-CoV-2 S1/S2 IgG were evaluated before vaccination (day 0 , first injection, time point [TP]0), after three weeks from vaccination (day 21, second injection, TP1), and two-weeks (day 35, TP2) and nine-weeks (day 84, TP3) post-booster. All participants were asked to provide nose and throat swabs for molecular determination of a SARS-CoV-2 infection at each of the first two TPs and in every case of clinical suspicion of COVID-19 or hospital access subsequently. A positive determination of anti SARS-CoV-2 $\mathrm{lgG}$ at basal was an exclusion criterion from the analysis. Anti SARSCoV-2 S1/S2 lgG determination was performed by the Liaison ${ }^{\circ}$ SARS-CoV-2 S1/S2 IgG assay (DiaSorin ", Saluggia, Italy), a quantitative chemiluminescent immunoassay approved by FDA. According to the manufacturers' technical manual, the result of a Liaison SARS-CoV-2 S1/S2 lgG test is positive with a signal of $15 \mathrm{AU} / \mathrm{mL}$ or higher [https://www.diasorin.com/en/immunodiagnostic-solutions/ clinical-areas/infectious-diseases/covid-19]. The most useful TI between last dose of anti-CD20 and vaccination for categorization purposes was identified at the cutoff value which best separated responders versus non-responders according to the Wilcoxon statistic (Torsten Hothorn [2017]. maxstat: Maximally Selected Rank Statistics. R package version 0.7-25. https://CRAN.R-project.org/ package $=$ maxstat).

From March 2 to April 20, 2021, 83 consecutive patients with B-NHL were vaccinated with BNT162b2 at our Institute. Fourteen patients were excluded from this analysis because of no

\footnotetext{
${ }^{1}$ Hematology Unit, Department of Research and Clinical Oncology, IRCCS Regina Elena National Cancer Institute, Rome, Italy. ${ }^{2}$ Microbiology and Virology Unit, Dermatological

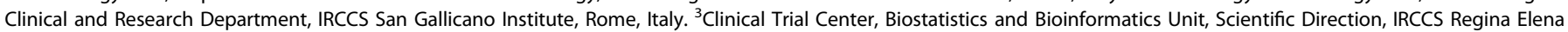

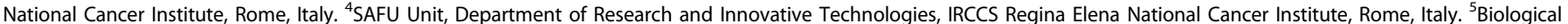

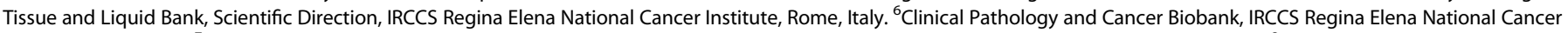

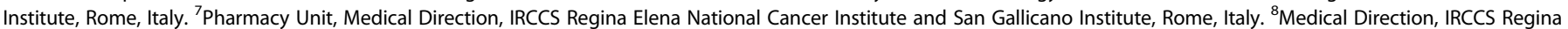

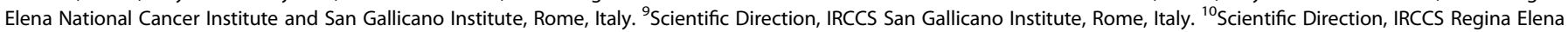

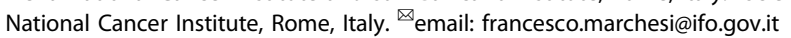

Received: 15 June 2021 Revised: 1 September 2021 Accepted: 7 September 2021

Published online: 20 September 2021 


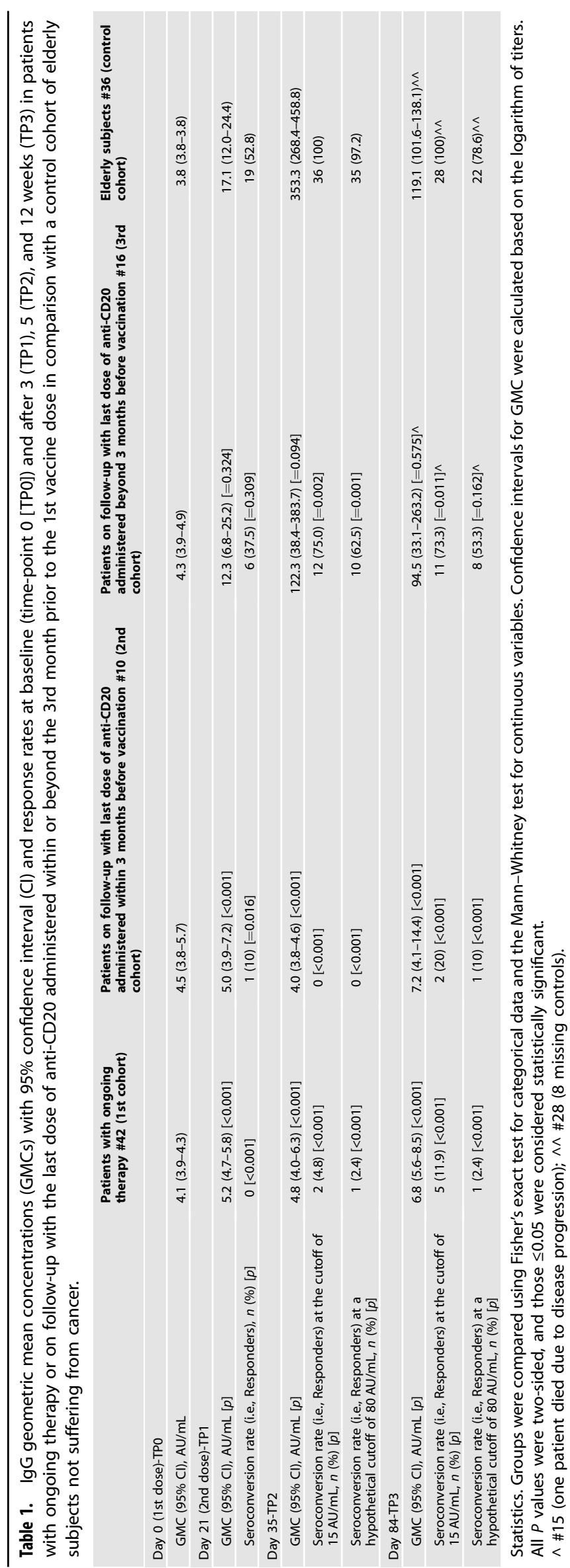

exposition to an anti-CD20 $(n=3)$, refusal to participate $(n=5)$, missing samples $(n=6)$. Of the remaining 69 patients, 1 was excluded because of severe COVID-19 diagnosed between the 1st and 2nd vaccine dose; in this patient the SARS-CoV-2 genome was directly obtained from nasopharyngeal swab fluid by nextgeneration sequencing (Illumina COVIDSeq Test on a Nextseq 500, Illumina Inc., San Diego, CA, USA); the lineage assigned by the Phylogenetic Assignment of Named Global Outbreak Lineages web-based software was B.1.1.7 with genome coverage of $99.76 \%$ (Global Initiative on Sharing All Influenza Dataset ID: EPI_ISL_2245016). The characteristics of the 68 study' patients are detailed in supplement data (Supplementary Table 1). The control cohort was represented by 36 subjects not suffering from cancer with a median age of 81 years (range 79-87) whose gender was female in $50 \%$ of cases. As detailed in supplement data (Supplementary Table 2 and Supplementary Fig. 1), a TI between the last anti-CD20 administration and the 1st vaccine dose at the cutoff of three months was identified as the strongest predictor of response to BNT162b2. Of the 52 pooled patients with ongoing therapy ( $n=42$, 1st cohort) or on follow-up with an anti-CD20vaccination $\mathrm{Tl}$ within three months ( $n=10,2$ nd cohort), 2 were responsive $(3.8 \%)$ compared to 12 out of $16(75.0 \%)$ on follow-up with a TI beyond three months (3rd cohort; $p<0.001$ ). Out of 2 responsive patients on active treatment, one was on zandelisib (female, 68 years, with follicular lymphoma in 3rd line of therapy, who received the 1 st dose of vaccine 87 months after the last dose of rituximab, $194 \mathrm{AU} / \mathrm{mL}$ at TP2) and the other on R-DHAOX (male, 67 years, with diffuse large B-cell lymphoma in 2nd line of therapy, who received the 1 st dose of vaccine 19 days after the dose of rituximab of the 1 st salvage cycle, 37.2 AU/mL at TP2).

We compared the serological response to BNT162b2 vaccine at 3,5 , and 12 weeks in terms of IgG geometric mean concentration (GMC) and response rate between the control group and the three different cohorts of patients (Table 1).

On TP1, no statistical difference was observed between the 3rd cohort of patients and the control cohort. The other two cohorts of patients did not respond at all with the exception of only one patient who seroconverted just above the cutoff of $15 \mathrm{AU} / \mathrm{mL}$ (17.8), but whose titer dropped to 3.8 at 5 weeks.

On TP2, antibody production increased in the 3rd group of patients, though at a lesser extent than that found in elderly controls, reaching a GMC of $122.3 \mathrm{AU} / \mathrm{mL}$ as compared to $353.3 \mathrm{AU} / \mathrm{mL}$ seen in elderly controls $(p=0.094)$. A seroconversion rate of $100 \%$ was found in elderly controls (36/36) while 12/16 (75.0\%) individuals of the 3rd group of patients seroconverted $(p=0.002)$. The other two groups of patients did not show any detectable response in the majority of individuals, with the exception of the two patients from the 1st group who have been previously described.

On TP3, a decay of antibody titer with a loss of GMC of around $30 \%$ vs TP2 was observed in patients of the 3rd group, less than that observed in the control group ( 60\%). Titer kinetics was flat among patients of the 1st and 2nd group [9].

Figure 1 shows the IgG GMCs on TP0, TP1, TP2, and TP3. Overall, the degree of the immune response elicited by vaccination largely depends upon the timing of anti-CD20 administration, and even for patients with $>3$ months of separation from the last dose of antiCD20, it is possible to observe a deeply depressed antibody response.

Our data confirm that the response to the BNT162b2 should be considered null during treatment with anti-CD20 moAbs, becoming present, though suboptimal, starting from the 3rd month after the last dose of anti-CD20.

Why in the face of previously published ECIL guidelines have we decided to proceed with the vaccination in a such unfavorable context? The answer is that, under the pressure of the pandemic, we have given up following the indication of science to take refuge behind the indications of the National Authorities. It is clear that no national vaccination plan to combat the pandemic could have foreseen all possible situations for all possible vulnerable categories 


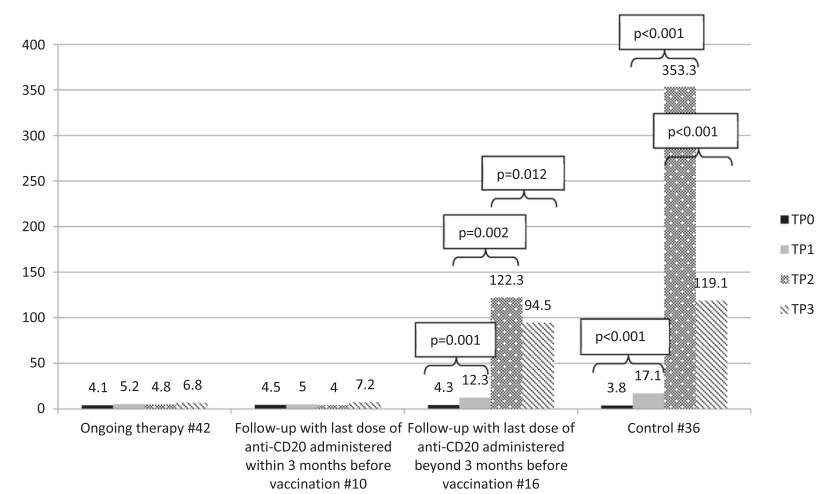

Fig. 1 IgG geometric mean concentrations on TP0, TP1, TP2, and TP3 in the cohorts, AU/mL. TPO is day of 1st dose, TP1 is day of 2nd dose (3rd week after 1st dose), TP2 is 5th week after 1st dose, TP3 is 12 th week after 1 st dose. To evaluate the effect of the second dose of vaccine and of IgG decay kinetics in the groups, the Wilcoxon test was used for comparing geometric mean concentration (GMC) between TP1, TP2, and TP3.

of patients. It is equally clear that we had to take responsibility for the decision to vaccinate or not these patients, on one hand considering the underlying disease and the ongoing or recent antiCD20 treatment, on the other with the awareness of being under the sword of Damocles for any litigations in the event of SARS-Cov-2 infection and death of patients who had to be vaccinated according to the indications of the national vaccination plan.

Second, authorizing a second round of vaccination for these unresponsive patients previously exposed to an anti-B-cell treatment is an issue that will need to be addressed by HA soon. In this regard, our findings provide some elements to help define the right timing for vaccination after an anti-CD20-based therapy. Our data suggest that, in patients with B-NHL, the TI required between the last dose of anti-CD20 and vaccination is of at least three months. This is a less extended time than that suggested by other authors who indicate a $\mathrm{TI}$ of at least 12 months after anti-CD20 therapy [1,5], and less than the six months indicated by the ECIL 7 guidelines [7]. The indications as well as the more appropriate schedule for additional vaccine boosters remain also to be established [10].

The limited observation period and the absence of concomitant investigations on cellular responses do not allow to draw conclusions on two questions which remain unanswered, namely whether responding patients actually develop true protection from SARS-CoV-2 infection and how long this protection extends over time, and, strictly linked to the latter, whether a more robust cellular immune response might be present in patients displaying a limited or absent antibody response.

\section{REFERENCES}

1. Herishanu Y, Avivi I, Aharon A, Shefer G, Levi S, Bronstein Y, et al. Efficacy of the BNT162b2 mRNA COVID-19 vaccine in patients with chronic lymphocytic leukemia. Blood. 2021;137:3165-73. https://doi.org/10.1182/blood.2021011568.

2. Roeker LE, Knorr DA, Thompson MC, Nivar M, Lebowitz S, Peters N, et al. COVID19 vaccine efficacy in patients with chronic lymphocytic leukemia. Leukemia. 2021 May 13:1-3. https://doi.org/10.1038/s41375-021-01270-w.

3. Pimpinelli F, Marchesi F, Piaggio G, Giannarelli $D$, Papa E, Falcucci $P$, et al. Fifthweek-immunogenicity and safety of anti-SARS-CoV-2 BNT162b2 vaccine in patients with multiple myeloma and myeloproliferative malignancies on active treatment: preliminary data from a single Institution. J Hematol Oncol. 2021;14:81. https://doi.org/10.1186/s13045-021-01090-6.

4. Perry C, Luttwak E, Balaban R, Shefer G, Morales MM, Aharon A, et al. Efficacy of the BNT162b2 mRNA COVID-19 vaccine in patients with B-cell non-Hodgkin lymphoma. Blood Adv. 2021;5:3053-61. https://doi.org/10.1182/bloodadvances.2021005094.
5. Gurion R, Rozovski U, Itchaki G, Gafter-Gvili A, Leibovitch C, Raanani P, et al. Humoral serologic response to the BNT162b2 vaccine is abrogated in lymphoma patients within the first 12 months following treatment with anti-CD2O antibodies. Haematologica. 2021 Jul 29. https://doi.org/10.3324/haematol.2021.279216.

6. Ghione P, Gu JJ, Attwood K, Torka P, Goel S, Sundaram S, et al. Impaired humoral responses to COVID-19 vaccination in patients with lymphoma receiving B-cell directed therapies. Blood. 2021 Jun 29:2021012443. https://doi.org/10.1182/ blood.2021012443.

7. Mikulska M, Cesaro S, de Lavallade H, Di Blasi R, Einarsdottir S, Gallo G, et al. Vaccination of patients with haematological malignancies who did not have transplantations: guidelines from the 2017 European Conference on Infections in Leukemia (ECIL7). Lancet Infect Dis. 2019;19:e188-99.

8. Vijenthira A, Gong I, Betschel SD, Cheung M, Hicks LK. Vaccine response following anti-CD20 therapy: a systematic review and meta-analysis of 905 patients. Blood Adv. 2021;5:2624-43. https://doi.org/10.1182/bloodadvances.2021004629.

9. Marchesi F, Pimpinelli F, Sperandio E, Papa E, Falcucci P, Pontone M, et al. Twelveweeks kinetics of anti-SARS-CoV-2 antibodies in different haematological cancers after vaccination with BNT162b2. Br J Haematol. 2021. https://doi.org/10.1111/ bjh.17821.

10. Besson C. It is time to adapt anti-CD20 administration schedule to allow efficient anti-SARS-Cov-2 vaccination in patients with lymphoid malignancies. Haematologica. 2021 Jul 29. https://doi.org/10.3324/haematol.2021.279457.

\section{ACKNOWLEDGEMENTS}

This work was supported in part by a grant provided by Istituto Soka Gakkai on an $8 \times 1000$ fund for the project COMETA coordinated by Prof. Gennaro Ciliberto. Special thanks to I. F. O.-COVID-19-Team.

\section{AUTHOR CONTRIBUTIONS}

FM gave a major contribution to construct dataset and to write and revise the paper. FP implemented the laboratory platforms in order to carry out the study, performed all serological and molecular tests, validated and interpreted the results and signed the reports. DG carried out statistical analysis. LR coordinated the collection of peripheral blood mononuclear cells and serum. EP and PF collected the signed informed consents and clinical data for the construction of the dataset. MP contributed to perform serological and molecular tests. EGDD contributed to perform serological and molecular tests and to validate the results. SDM, VL, and CM were in charge of preservation of peripheral blood mononuclear cells and serum at biological bank. LC and IC had responsibility to coordinate lymphoma diagnosis process at laboratory level. ALM organized acquisition, storage, distribution and administration of vaccine. CV was the case manager who organized the vaccination lists and took the blood samples. DR, FP, AR, FP SG interviewed the patients as for side effects, collected signed informed consents and clinical data for the construction of the dataset. ODB managed the control cohort. BV organized the vaccination slots and allocated human resources for the conduction of the study. AMo conceived and projected the study. GC conceived and projected the study and revised the paper. FE handled laboratory work-flow, and contributed to conceive and to write the study protocol, and revised the paper. AMe contributed to project the study, to construct the dataset, to interpret the data and to perform the statistical analysis, and wrote the paper. All the authors read and approved the final paper.

\section{COMPETING INTERESTS}

The authors declare no competing interests.

\section{ADDITIONAL INFORMATION}

Supplementary information The online version contains supplementary material available at https://doi.org/10.1038/s41375-021-01418-8.

Correspondence and requests for materials should be addressed to Francesco Marchesi.

Reprints and permission information is available at http://www.nature.com/ reprints

Publisher's note Springer Nature remains neutral with regard to jurisdictional claims in published maps and institutional affiliations. 